\title{
Prognostic significance of the angiopoietin-2/ angiopoietin-1 and angiopoietin-1/Tie-2 ratios for early sepsis in an emergency department
}

\author{
Yingying Fang, Chunsheng Li*, Rui Shao, Han Yu, Qing Zhang and Lianxing Zhao
}

\begin{abstract}
Introduction: This study was performed to assess the early diagnostic, risk stratification, and prognostic value of the angiopoietin-2/angiopoietin-1 ratio (Ang-2/Ang-1) and angiopoietin-1/tyrosine kinase with immunoglobulin-like loop epidermal growth factor homology domain 2 ratio (Ang-1/Tie-2) and to compare these factors with procalcitonin (PCT) and the Mortality in Emergency Department Sepsis (MEDS) score in patients with early sepsis in the emergency department (ED).

Methods: Consecutive patients with sepsis $(n=440)$ were enrolled in this study. They fulfilled the systemic inflammatory response syndrome (SIRS) criteria and were admitted to the ED of Beijing Chao-yang Hospital between August 2014 and February 2015. The control group consisted of 55 healthy blood donors. The patients were categorized into four groups: SIRS, sepsis, severe sepsis, and septic shock. Serum Ang-1, Ang-2, Tie-2, and PCT were measured, and the MEDS score was calculated upon ED arrival. The prognostic values of Ang-2/Ang-1, Ang-1/Tie-2, Ang-1, Ang-2, and Tie-2 were compared with the PCT and MEDS scores. All patients were followed for 28 days.
\end{abstract}

Results: Upon admission, the median levels of the serum Ang-2 level and Ang-2/Ang-1 ratio increased and the serum Ang-1 levels and Ang-1/Tie-2 ratios decreased with the severity of sepsis. The areas under the receiver operating characteristic curves of the Ang-2/Ang-1 and Ang-1/Tie-2 ratios were greater than those of the Ang-1, Ang-2, and PCT levels and MEDS scores in the diagnosis and prediction of 28-day mortality due to sepsis. Ang-2/Ang-1 was significantly higher and Ang-1/Tie-2 was significantly lower in nonsurvivors than in survivors at the 28-day follow-up examination. Ang-2/Ang-1, Ang-1/Tie-2, and MEDS score were found to be independent predictors of 28-day mortality in patients with sepsis. The levels of serum Ang-1, Ang-2, and Tie-2 were positively correlated with each other. The ratios of Ang-2/Ang-1 and Ang-1/Tie-2 were positively and negatively correlated, respectively, with the MEDS score in every septic group.

Conclusions: The Ang-2/Ang-1 and Ang-1/Tie-2 ratios are valuable for risk stratification in patients with sepsis and are associated with the poor clinical outcome of early sepsis in the ED.

\section{Introduction}

Sepsis continues to be a very significant cause of mortality. Sepsis with multiple organ dysfunction syndrome (MODS) is the most catastrophic manifestation. In addition, patients who develop severe sepsis or septic shock have worse mortality than patients who do not develop sepsis [1, 2]. Effective management and resource allocation is difficult because of the inability to diagnose

\footnotetext{
* Correspondence: Icscyyy04@sina.com

Emergency Department, Beijing Chao-yang Hospital, Capital Medical University, 8 Worker's Stadium South Road, Beijing, Chao-yang District 100020, China
}

the severity and predict the high risk of sepsis. Although current biomarkers show great promise in indicating the severity of sepsis, the highly variable and nonspecific nature of the signs and symptoms of sepsis makes the prospect of single biomarker classification less valuable. Currently, it is of great significance to identify biomarkers and combine them with clinical scoring systems for risk stratification and evaluation of the prognosis of sepsis.

Among the complex mechanisms and heterogeneous nature of sepsis, widespread endothelial dysfunction plays an extremely important role in the severity of sepsis and 
sepsis-induced MODS [1, 3, 4]. The endothelium is a key target of sepsis-induced events, and the sepsis-activated vascular endothelium is responsible for the increase in luminal cell adhesion molecules, leukocyte recruitment, vasomotor tone alteration, microvascular thrombosis formation, and eventually diffuse capillary leakage $[4,5]$. One of the most important mechanisms activating endothelial cells during sepsis is the endothelium-specific angiopoietin (Ang) tyrosine kinase with the immunoglobulin-like loop epidermal growth factor domain (Tie) ligand-receptor system, which has a potential correlation with endothelial injury severity. Ang-1, Ang-2, and Tie-2 play different roles in mediating vascular quiescence and inflammation. Ang-1 promotes vessel stability, suppresses inflammation, and promotes endothelial cell survival by activating the Tie-2 receptor complex [6-8], whereas Ang-2 destabilizes blood vessels, potentiates inflammation, and promotes proangiogenic effects, which result in vascular leakage and organ dysfunction by initially blocking the Tie- 2 receptor $[6,9,10]$. The Tie-2 receptor is expressed and activated throughout the quiescent adult endothelium [11], where it promotes microvascular barrier function and antiinflammation [12]. Investigators have investigated Ang-1 and Ang-2 in various studies as biomarkers of sepsis severity and mortality because of their roles in endothelial activation and their convenient measurement in the ED. Previous studies have shown that levels of Ang-1 and Ang-2 are clinically informative prognostic biomarkers of mortality in severe sepsis [13]. Ang-1 protects against organ dysfunction in animal models of sepsis [14], whereas Ang-2 is associated with sepsis severity and multiple organ dysfunction in sepsis in vitro [15] and in vivo [16]. However, in most of the previous studies, evidence for the use of the Ang-2/Ang-1 and Ang-1/ Tie-2 ratios in predicting the severity and high mortality in patients with early sepsis has been lacking. Given these considerations, the aim of our present study was to investigate the correlation of serum the Ang-2/Ang-1 and Ang-1/Tie-2 ratios with risk stratification and prognostic evaluation of various degrees of early sepsis compared with PCT levels and MEDS scores.

\section{Material and methods}

\section{Patients}

This study was approved by the human research ethics committee of Beijing Chao-yang Hospital affiliated with the Capital Medical University (Beijing, China), and signed written informed consent forms were obtained from patients upon ED admission. The biological specimens and clinical data sets were obtained from a prospective observational study of patients who had a suspected infection with two or more criteria of systemic inflammatory response syndrome (SIRS) and who were enrolled in the ED of Beijing Chao-yang Hospital, which had an average of
260,000 visits annually during the period between August 2014 and January 2015. The patient records were anonymous and deidentified before analysis. The exclusion criteria were (1) age $<18$ years old or $>80$ years old; (2) pregnancy or breastfeeding; (3) neutropenia, defined as $<1000$ neutrophils $/ \mathrm{mm}^{3}$; (4) HIV infection; (5) hypertension or constantly taking angiotensin-converting enzyme inhibitors, angiotensin II receptor antagonists, or renin inhibitors; (6) chronic intake of corticosteroids, defined as any daily oral intake of $1 \mathrm{mg} / \mathrm{kg}$ or more of equivalent prednisone for more than 1 month; (7) recent use of doses of unfractionated or low-molecular-weight heparin; and (8) lack of informed consent by the patients or their relatives.

A total of 440 patients who were followed for 28 days or until death were categorized on the basis of the 2001 Society of Critical Care Medicine/European Society of Intensive Care Medicine/American College of Chest Physicians/American Thoracic Society/Surgical Infection Society International Sepsis Definitions Conference and Surviving Sepsis Campaign guidelines $[17,18]$ into the following subgroups: SIRS $(n=107)$, sepsis $(n=176)$, severe sepsis $(n=84)$, and septic shock $(n=73)$. The control group included 55 healthy blood donors with no history or clinical evidence of acute or chronic disease. They were similar in age and sex to the experimental groups.

\section{Sample collection and measurements}

Most of the blood samples were obtained from blood drawn for routine diagnostic procedures, and no additional blood was obtained from the patients. Informed consent was provided by the patients or their firstdegree relatives and by healthy control subjects before blood collection. Within 15 min of arrival in the ED, a 5 -ml sample of blood was collected from patients and healthy donors in tubes containing ethylenediaminetetraacetic acid. The samples were immediately transferred to the local central laboratory. The serum was stored at $-80{ }^{\circ} \mathrm{C}$ until further measurement.

Samples were analyzed by researchers who were blinded to all patient data. Serum Ang-1, Ang-2, and Tie-2 were measured in available samples upon admission using an enzyme-linked immunosorbent assay (Abcam, Cambridge, MA, USA). PCT levels were measured using an enzymelinked fluorescence immunoassay with a miniVIDAS immunoassay analyzer (bioMérieux, Durham, NC, USA). All standards, controls, and test samples were assayed in duplicate. All measurements were performed according to the manufacturers' instructions.

\section{Calculation of the MEDS score and APACHE II score}

The MEDS score was calculated by summing the points of nine variables: terminal illness, tachypnea or hypoxia, septic shock, low platelet count, age $>65$ years, lower 
respiratory tract infection, nursing home resident, and altered mental status [19]. The Acute Physiology and Chronic Health Evaluation (APACHE) II score consisted of measurements of the body temperature, mean arterial pressure, heart rate, respiratory rate, oxygenation, arterial $\mathrm{pH}$, sodium, potassium, creatinine, hematocrit, white blood cell count, Glasgow Coma Scale score, age, chronic diseases, and surgical status, with a total possible score between 0 and 71 [20].

\section{Statistical analyses}

These results were presented as the mean standard deviation or median range (interquartile range). Nonnormal distribution of continuous variables was tested using the Kolmogorov-Smirnov test and Mann-Whitney $U$ test. In patients, differences between survivors and nonsurvivors were also compared using a nonparametric two-sided Mann-Whitney $U$ test. Comparisons between groups are illustrated with box plot graphics, where the dotted line indicates the median, the box represents the 25th75th percentiles, the horizontal lines show the minimum and maximum values of the calculated nonoutlier values, and the open circles indicate outlier values. To determine independent predictors of 28-day mortality, logistic regression analysis was performed while adjusting for age, sex, type of infection, and infection sites. A receiver operating characteristic (ROC) curve was used to assess the accuracy of the variables in prediction of 28-day mortality. In addition, the comparisons of areas under the ROC curves (AUCs) were analyzed using MedCalc version 11.6 software (MedCalc Software, Ostend, Belgium). Furthermore, the optimal cutoff value for Ang-2/Ang-1 and Ang-1/Tie-2 ratios to differentiate between survivors and nonsurvivors was determined empirically from the ROC curve using the Youden index [21]. Two-sided $p$ values of 0.05 were considered statistically significant. Confidence intervals (CIs) were reported at $95 \%$. Kaplan-Meier survival curves were constructed for patients to examine the prognostic value of the Ang-2/Ang-1 and Ang-1/Tie-2 ratios according to their levels at ED admission. The logrank test was used to compare survival rates between groups. Finally, Spearman's rank correlation coefficient was calculated to describe correlations between Ang-1, Ang-2, and Tie-2 levels, as well as Ang-2/Ang-1 ratio, Ang-1/Tie-2 ratio, and MEDS score. All of the data were analyzed using IBM SPSS 19.0 software (IBM, Armonk, NY, USA).

\section{Results}

\section{Patient characteristics}

A total of 440 patients and 55 healthy controls were enrolled in the study and the recruitment of patients into the study is shown in Fig. 1. There were no significant differences in age, sex, heart rate (HR), ratio of partial pressure arterial oxygen to fraction of inspired oxygen, infections between the five groups (healthy controls, SIRS, sepsis, severe sepsis, and septic shock). There were significant differences between groups in the APACHE II and MEDS scores $(p<0.0001)$. The basic characteristics, mediators and infections of the enrolled subjects are listed in Tables 1 and 2.

\section{Comparison of median Ang-1, Ang-2, and Tie-2 levels, Ang-2/Ang-1 ratio, Ang-1/Tie-2 ratio, and MEDS score} Table 2 shows, for each study group, the median Ang-1, Ang-2, and Tie-2 levels; Ang-2/Ang-1, and Ang-1/Tie-2 ratios; the PCT level; and the MEDS score. Figure 2 displays the Ang-1, Ang-2, and Tie-2 levels in each group. Serum Ang-1, Ang-2, Tie-2, and PCT and the MEDS score at ED admission were significantly different among the groups. Compared with the healthy control group, the Ang-2, Tie-2, PCT level, and MEDS score were significantly higher, and Ang-1 level was lower, in patients with SIRS, sepsis, severe sepsis, and septic shock $(p<0.0001)$. Interestingly, the Ang-2/Ang-1 and Ang-1/Tie-2 ratios were markedly different in sepsis, severe sepsis, and septic shock compared with the control group $(p<0.0001)$.

\section{Ang-2/Ang-1 and Ang-1/Tie-2 ratios in survivors and nonsurvivors}

The Ang-2/Ang- 1 ratio was higher in survivors than in nonsurvivors, whereas the Ang- $1 / \mathrm{Tie}-2$ ratio was lower in survivors than in nonsurvivors $(p<0.0001)$ (Fig. 3).

\section{Value of the Ang-2/Ang-1 and Ang-1/Tie-2 ratios and MEDS scores for predicting 28-day mortality}

The AUC of Ang-2/Ang-1 ratio for predicting 28-day mortality in patients with sepsis was 0.845 higher than PCT (0.732) and Ang-1 and Ang-2 levels (0.778 and 0.794 , respectively) (all $p<0.05$ ). The AUC of Ang- $1 /$ Tie- 2 ratio for predicting 28-day mortality in patients with sepsis was 0.808 higher than PCT $(0.732)(p<0.05)$. The AUC of Ang-2/Ang-1 ratio for predicting 28-day mortality was 0.845 , higher than that of MEDS score (0.826), but was not statistically significant $(p>0.05)$. The AUC of Ang- $2 /$ Ang-1 ratio in combination with the MEDS score was 0.857 , which was statistically significant compared with MEDS score $(0.826)(p<0.05)$. The AUC of Ang- $1 /$ Tie-2 ratio in combination with MEDS score was higher than MEDS score alone (0.844 vs. $0.826 ; p<0.05)$.

The AUC for a combination of Ang-2/Ang-1 ratio, MEDS score, and PCT was significantly higher than that for PCT combined with MEDS score (0.900 vs. $0.829 ; p<$ 0.01). The AUC for a combination of Ang- $1 /$ Tie- 2 ratio, MEDS score, and PCT was also significantly higher than that of PCT combined with MEDS score (0.894 vs. $0.829 ; p<0.05)$. Among the prognostic factors studied, the best rate for prediction of 28-day mortality was 


\section{CONSORT 2010 Flow Diagram}

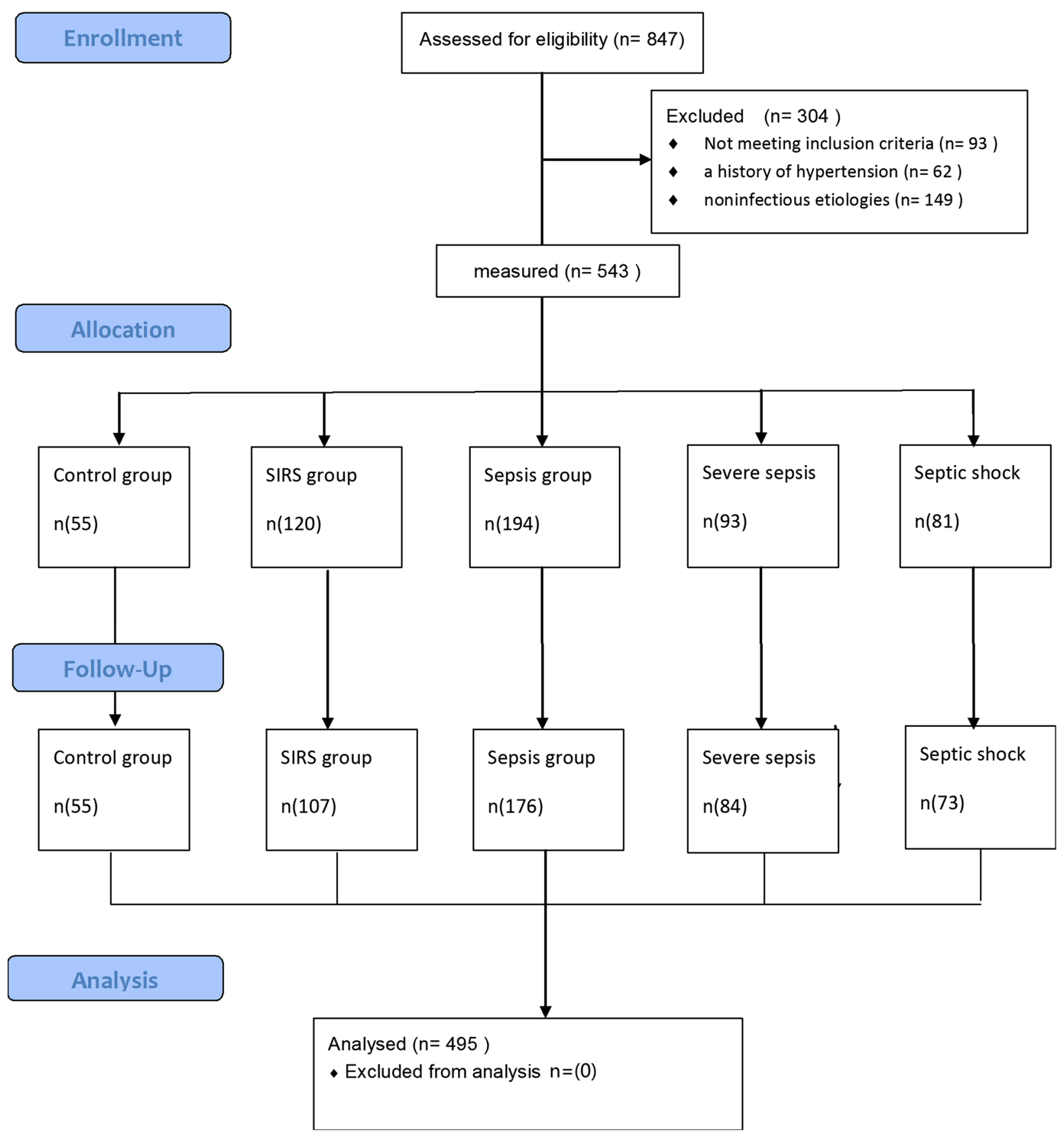

Fig. 1 CONSORT flow diagram of the study. SIRS systemic inflammatory response syndrome

for a combination of Ang-2/Ang-1 and Ang-1/Tie-2 ratios, MEDS score, and $\mathrm{PCT}$, which was significantly higher than for each parameter alone $(p<0.01)$. All of the data were analyzed using MedCalc version 11.6 software. The ROC curves of these biomarkers and MEDS scores for predicting 28-day mortality among the five groups are shown in Fig. 4.
Using an Ang-2/Ang-1 ratio cutoff value of 1.94 for predicting 28-day mortality in patients with sepsis, the sensitivity was $79.7 \%$, the specificity was $81.3 \%$, the positive predictive value (PPV) was $78.4 \%$, the negative predictive value (NPV) was $82.5 \%$, the positive likelihood ratio $(\mathrm{LR}+)$ was 3.98 , and the negative likelihood ratio (LR-) was 0.31 . Using an Ang-1/Tie-2 ratio cutoff 
Table 1 Basic characteristics of the study population

\begin{tabular}{|c|c|c|c|c|c|c|}
\hline Characteristics & Control & SIRS & Sepsis & Severe sepsis & Septic shock & $p$ value \\
\hline Number of subjects & 55 & 107 & 176 & 84 & 73 & \\
\hline \multicolumn{7}{|l|}{ Demographic data } \\
\hline Age (yr) & $61(54-71)$ & $68(56-76)$ & $71(59-78)$ & $74(61-78)$ & $75(65-78)$ & 0.202 \\
\hline Male (\%) & 54.5 & 52.6 & 61.0 & 60.3 & 58.1 & 0.831 \\
\hline \multicolumn{7}{|l|}{ Physiological data } \\
\hline $\mathrm{MAP}(\mathrm{mmHg})$, mean $\pm \mathrm{SD}$ & $100 \pm 19$ & $110 \pm 21$ & $90 \pm 15$ & $89 \pm 13$ & $64 \pm 14$ & 0.042 \\
\hline $\mathrm{HR}$ (beats/min), mean $\pm \mathrm{SD}$ & $79 \pm 22$ & $103 \pm 21$ & $99 \pm 15$ & $105 \pm 23$ & $105 \pm 23$ & 0.339 \\
\hline $\mathrm{PaO}_{2} / \mathrm{FIO}_{2}$ ratio & $306(258-379)$ & $300(255-369)$ & $293(245-363)$ & $235(130-295)$ & $213(103-265)$ & 0.098 \\
\hline APACHE II score & 0 & $6.00(3.00-8.00)$ & $7.00(6.07-9.00)$ & $15.5(10.0-22.0)$ & $21.0(16.1-29.0)$ & $<0.0001$ \\
\hline MEDS score & 0 & $5.00(3.00-6.00)$ & $7.00(6.00-8.00)$ & $19.0(18.0-22.0)$ & $25.0(24.0-26.0)$ & $<0.0001$ \\
\hline
\end{tabular}

Abbreviations: SD standard deviation, SIRS systemic inflammatory response syndrome, APACHE Acute Physiology and Chronic Health Evaluation, MEDS Mortality in Emergency Department Sepsis, MAP mean arterial pressure, $\mathrm{HR}$ heart rate, $\mathrm{PaO}_{2} / \mathrm{FiO}_{2}$ ratio of partial pressure arterial oxygen to fraction of inspired oxygen

value of 30.0 for predicting 28 -day mortality in patients with sepsis, the sensitivity was $73.0 \%$, the specificity was 82.4 \%, the PPV was $79.4 \%$, the NPV was $77.9 \%$, the $\mathrm{LR}+$ was 4.15 , and the LR- was 0.33 . The detailed results are presented in Tables 3 and 4.

\section{Ang-2/Ang-1 ratio and MEDS score as independent predictors of 28-day mortality}

Age, sex, Ang-1, Ang-2, and Tie-2 levels, Ang-2/Ang-1 ratio, Ang-1/Tie-2 ratio, PCT, and MEDS score were included in a multivariate logistic regression model to determine the independent predictors of 28-day mortality. Ang-2/Ang-1 $(\beta=-0.013$, odds ratio $[\mathrm{OR}]=0.987, p<$ $0.0001)$, Ang- $1 /$ Tie-2 $(\beta=-6.553$, OR $=0.011, p=0.004)$, and MEDS score $(\beta=0.167$, OR $=1.182, p<0.0001)$ were found to be independent predictors of 28-day mortality in patients with sepsis (Table 5).

\section{Survival}

Using cutoff values determined by ROC curves, KaplanMeier survival curves were established. Figure 5a illustrates the Kaplan-Meier curves of 28-day survival stratified by

Table 2 Mediators and infections of the study population

\begin{tabular}{|c|c|c|c|c|c|c|}
\hline & Control & SIRS & Sepsis & Severe sepsis & Septic shock & $p$ value \\
\hline Number of subjects & 55 & 107 & 176 & 84 & 73 & \\
\hline \multicolumn{7}{|l|}{ Mediator levels } \\
\hline PCT (ng/ml) & $0.11(0.09-0.13)$ & $0.64(0.56-0.71)$ & $1.20(1.09-6.67)$ & $7.97(3.06-12.3)$ & $13.2(1.21-33.0)$ & $<0.0001$ \\
\hline Ang-1 (ng/ml) & $9.54(7.56-10.89)$ & $9.11(5.67-11.2)$ & $7.57(6.78-8.75)$ & $5.32(4.56-6.24)$ & $3.06(0.99-7.45)$ & $<0.0001$ \\
\hline Ang-2 (ng/ml) & $3.41(1.45-6.23)$ & $5.42(5.18-5.91)$ & $13.0(12.0-6.24)$ & $18.7(12.1-19.4)$ & $15.7(11.4-27.8)$ & $<0.0001$ \\
\hline Tie-2 (ng/ml) & $107.9(92.1-129.3)$ & 113.0 (99.8-189.9) & 174.7 (134.2-220.4) & $272.1(176.5-358.4)$ & 311.9 (149.9-415.2) & $<0.0001$ \\
\hline Ang-2/Ang-1 ratio & $0.49(0.16-0.61)$ & $0.73(0.51-0.91)$ & $1.73(1.37-2.00)$ & $3.42(1.93-4.15)$ & $5.14(1.50-18.95)$ & $<0.0001$ \\
\hline Ang-1/Tie-2 ratio $\left(\times 10^{3}\right)$ & $82.4(68.3-98.0)$ & $63.0(40.6-84.4)$ & $43.1(34.9-62.2)$ & $17.0(11.5-28.8)$ & $8.7(1.8-41)$ & $<0.0001$ \\
\hline \multicolumn{7}{|l|}{ Infection data (n) } \\
\hline \multirow[t]{3}{*}{ Respiratory (lung) } & & AECOPD (77) & Pneumonia (121) & Pneumonia (60) & Pneumonia (50) & 0.564 \\
\hline & & Asthma (9) & & & & \\
\hline & & $\mathrm{PE}(2)$ & & & & \\
\hline Intra-abdominal sites & & Pancreatitis (7) & $|A|(24)$ & $\mid \mathrm{Al}(14)$ & $|A|(15)$ & 0.558 \\
\hline Cerebral & & Stroke (8) & Meningitis (15) & Meningitis (5) & Meningitis (6) & 0.695 \\
\hline Urinary & & & Pyelonephritis (10) & Pyelonephritis (5) & Pyelonephritis (2) & 0.754 \\
\hline Other & & DKA (4) & Skin/soft tissue infection (6) & & & \\
\hline 28-day mortality, n (\%) & 0 & $15(14.0)$ & $32(18.2)$ & $36(42.9)$ & $45(61.6)$ & $<0.0001$ \\
\hline Missing data, n (\%) & 0 & $13(10.8)$ & $18(9.3)$ & $9(9.7)$ & $8(9.9)$ & 0.770 \\
\hline
\end{tabular}

Abbreviations: SIRS systemic inflammatory response syndrome, PCT procalcitonin, Ang angiopoietin, Tie-2 tyrosine kinase with immunoglobulin-like loop epidermal growth factor domain 2, AECOPD acute exacerbations of chronic obstructive pulmonary disease, $P E$ pulmonary embolism, $R /$ respiratory infection, $I A /$ intraabdominal infection, DKA diabetic ketoacidosis 

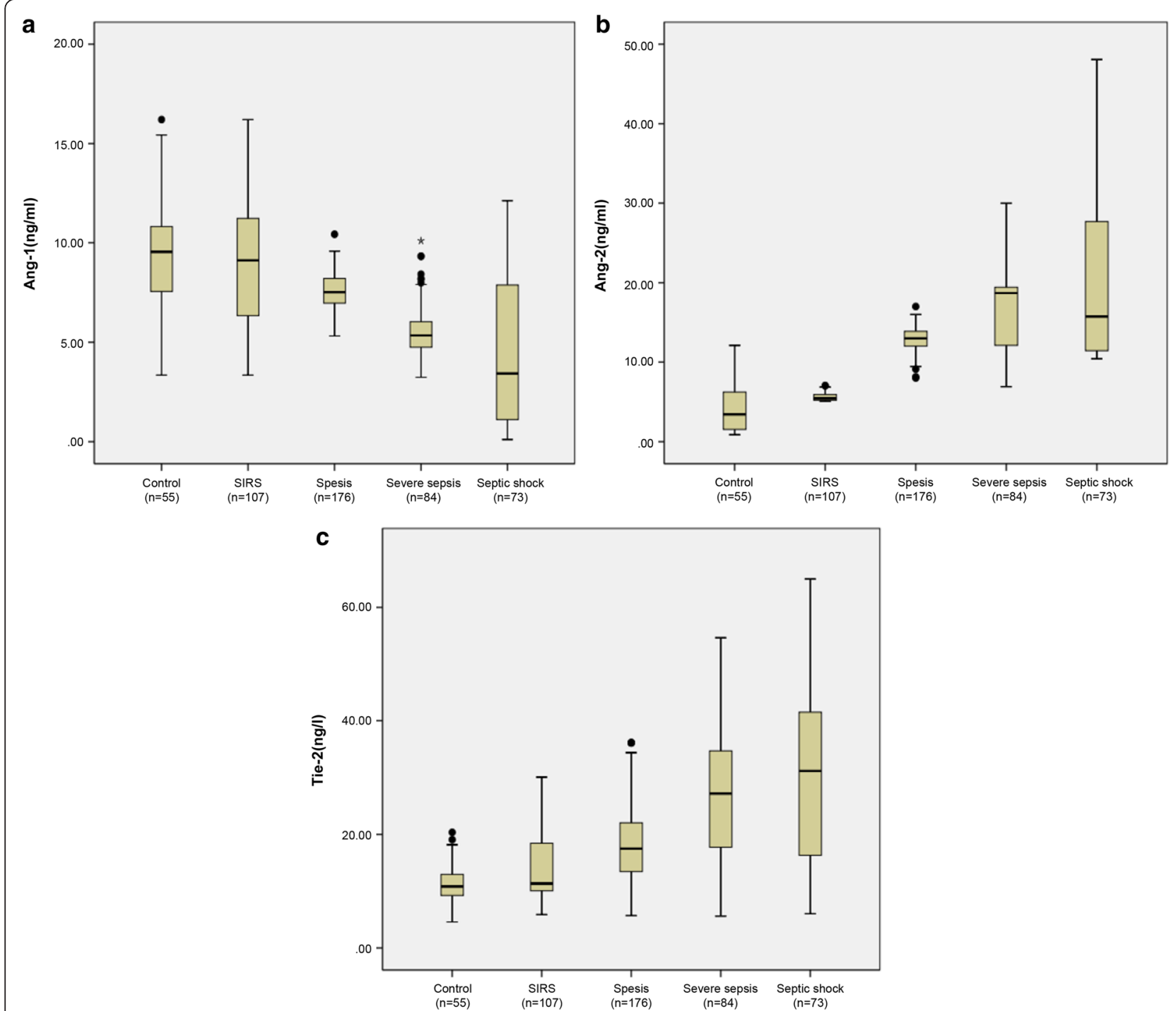

Fig. 2 The serum levels of Ang-1 (a), Ang-2 (b), and Tie-2 (c) in healthy control individuals and in patients with systemic inflammatory response syndrome, sepsis, severe sepsis, and septic shock upon emergency department admission. Ang angiopoietin, SIRS systemic inflammatory response syndrome, Tie-2 tyrosine kinase with immunoglobulin-like loop epidermal growth factor homology domain 2

the admission levels of the Ang-2/Ang-1 ratio above and below 1.94. The log-rank test confirmed the statistical significance of Ang-2/Ang-1 (log-rank $=94.42 ; p<0.0001$ ). Similarly, Kaplan-Meier survival analysis also showed that patients with sepsis with a serum Ang-1/Tie-2 ratio lower than 30.0 had a higher probability of survival at 28 days, as shown in Fig. 5b (log-rank = 100.48; $p<0.0001)$.

\section{Correlation of serum levels of Ang-1 and Ang-2 with serum Tie-2 levels and Ang-2/Ang-1 ratio, Ang-1/Tie-2 ratio with MEDS score}

Spearman's correlation analysis of Ang-1 and Ang-2 with Tie-2 showed correlation coefficients of $0.574,-0.613$, and 0.637 , respectively (all $p<0.0001$ ). The correlation coefficients between Ang-2/Ang-1, Ang-1/Tie-2, and MEDS score were 0.527 and -0.555 , which suggested a positive and negative correlation, respectively (all $p<0.0001$ ).

\section{Discussion}

It is important to identify useful biomarkers to improve early diagnosis and risk stratification, particularly in the early stages of sepsis. In our study, patients with sepsis presented with significantly decreased serum Ang-1 levels and Ang-1/Tie-2 ratios and markedly elevated serum Ang- 2 and Tie-2 levels and Ang-2/Ang- 1 ratios measured at admission [13, 22, 23]. The Ang-2/Ang-1 and Ang-1/ 

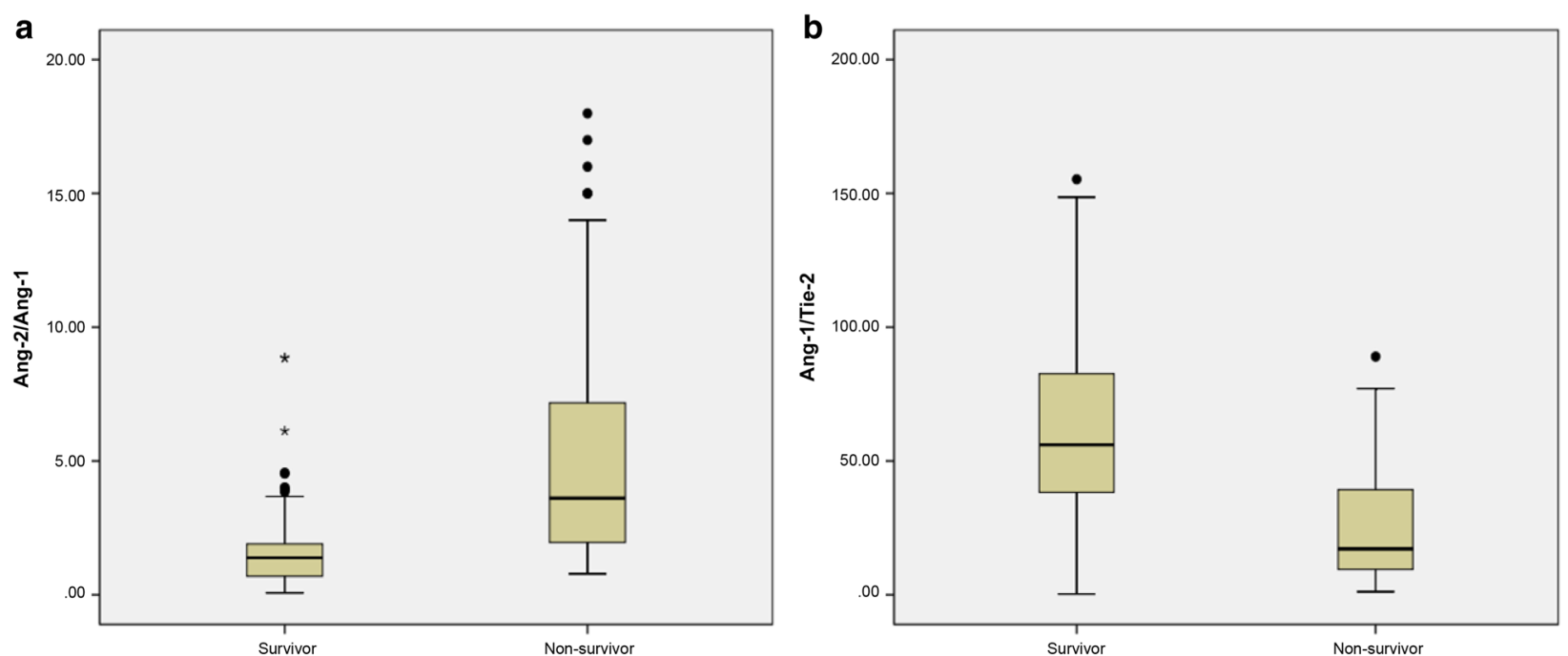

Fig. 3 Ang-2/Ang-1 (a) and Ang-1/Tie-2 (b) ratios at admission in survivor and nonsurvivor groups of patients with sepsis at 28-day follow-up. Lines denote he median values, boxes represent 25 th-75th percentiles, and whiskers indicate the range. Ang angiopoietin, Tie-2 tyrosine kinase with immunoglobulin-like loop epidermal growth factor homology domain 2
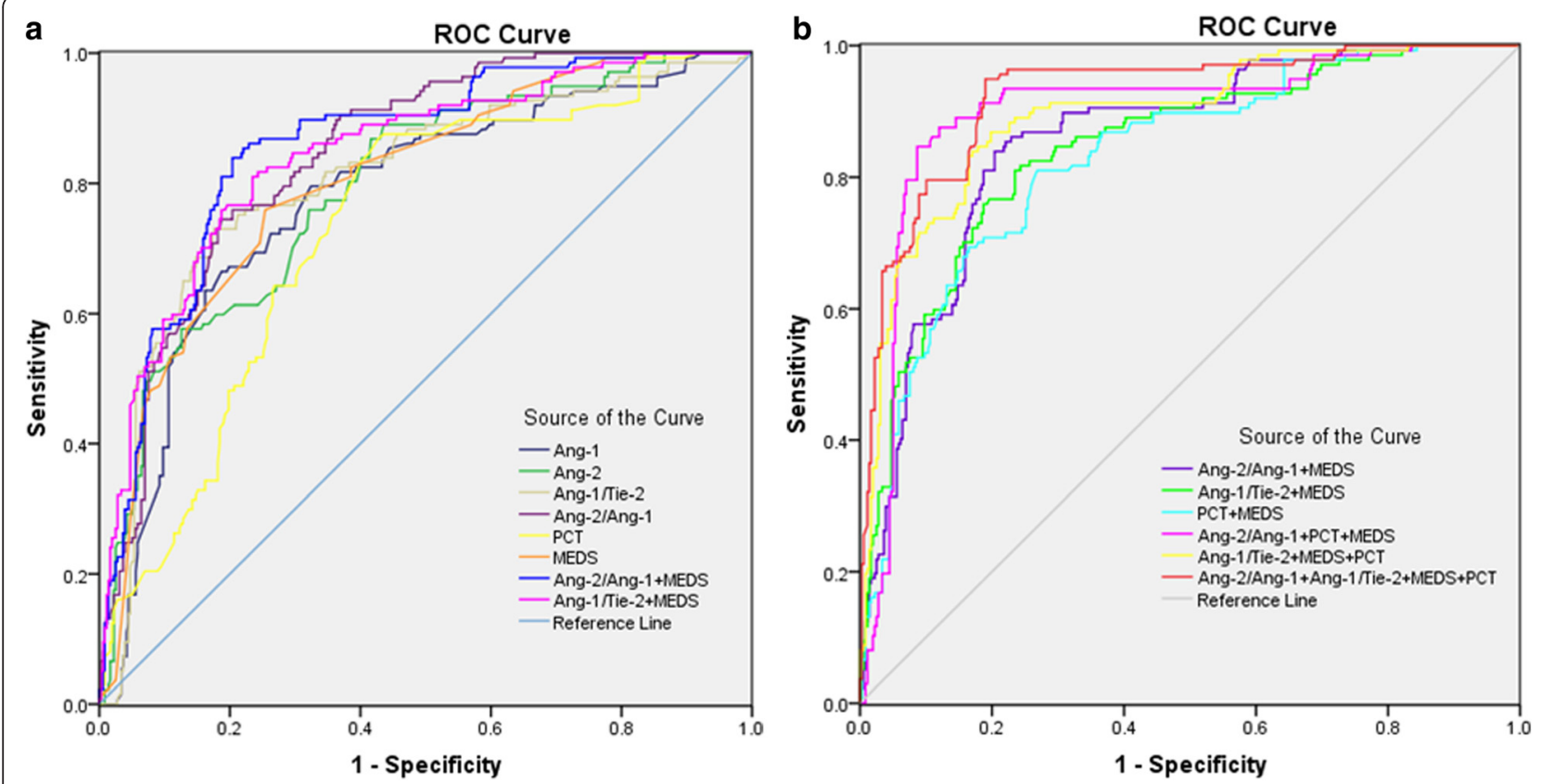

Fig. 4 ROC curves for predicting 28-day mortality in patients with sepsis. a AUCs for predicting 28-days mortality: Ang-2/Ang-1 ratio in combination with MEDS score $=0.857$ (blue line), Ang-1/Tie-2 ratio in combination with MEDS score $=0.844$ (pink line). $\mathbf{b}$ Similarly, AUCs for predicting 28-days mortality: Ang-2/Ang-1 ratio in combination with MEDS $=0.857$ (purple line), Ang-1/Tie-2 ratio in combination with MEDS score $=0.844$ (green line), PCT in combination with MEDS score $=0.829$ (blue line), a combination of Ang-2/Ang-1 ratio, MEDS score, and PCT $=0.900$ (pink line), a combination of Ang-1/Tie-1 ratio, MEDS score, and PCT = 0.894 (yellow line), a combination of Ang-2/Ang-1, Ang-1/Tie-1 ratios, MEDS score, and PCT $=0.925$ (red line). Ang angiopoietin, AUC area under the receiver operating characteristic curve, MEDS Mortality in Emergency Department Sepsis, PCT procalcitonin, ROC receiver operating characteristic, Tie-2 tyrosine kinase with immunoglobulin-like loop epidermal growth factor homology domain 2 
Table 3 AUCs for predicting 28-day mortality in patients with early sepsis

\begin{tabular}{|c|c|c|c|c|c|c|}
\hline & \multirow[t]{2}{*}{ Variable } & \multirow[t]{2}{*}{ AUC } & \multirow[t]{2}{*}{ Standard error } & \multirow[t]{2}{*}{$p$ value } & \multicolumn{2}{|l|}{$95 \% \mathrm{Cl}$} \\
\hline & & & & & Lower limit & Upper limit \\
\hline \multirow[t]{13}{*}{ 28-day mortality } & Ang-1 & 0.778 & 0.024 & 0 & 0.732 & 0.824 \\
\hline & Ang-2 & 0.794 & 0.018 & 0 & 0.750 & 0.837 \\
\hline & Tie-2 & 0.803 & 0.024 & 0 & 0.756 & 0.849 \\
\hline & Ang-2/Ang-1 & 0.845 & 0.018 & 0 & 0.810 & 0.880 \\
\hline & Ang-1/Tie-2 & 0.808 & 0.023 & 0 & 0.763 & 0.853 \\
\hline & PCT & 0.732 & 0.024 & 0 & 0.685 & 0.780 \\
\hline & MEDS score & 0.826 & 0.021 & 0 & 0.785 & 0.866 \\
\hline & Ang-2/Ang-1+MEDS score & 0.857 & 0.018 & 0 & 0.821 & 0.893 \\
\hline & Ang-1/Tie-2+MEDS score & 0.844 & 0.020 & 0 & 0.805 & 0.883 \\
\hline & PCT+MEDS score & 0.829 & 0.020 & 0 & 0.790 & 0.869 \\
\hline & Ang-2/Ang-1+PCT+MEDS score & 0.900 & 0.017 & 0 & 0.866 & 0.934 \\
\hline & Ang-1/Tie-2+PCT+MEDS score & 0.894 & 0.016 & 0 & 0.862 & 0.926 \\
\hline & Ang-2/Ang-1+Ang-1/Tie-2+PCT+MEDS score & 0.925 & 0.013 & 0 & 0.898 & 0.951 \\
\hline
\end{tabular}

Abbreviations: Ang angiopoietin, AUC area under the receiver operating characteristic curve, Cl confidence interval, MEDS Mortality in Emergency Department Sepsis, PCT procalcitonin, Tie-2 tyrosine kinase with immunoglobulin-like loop epidermal growth factor homology domain 2

Tie-2 ratios are considered to be valuable biomarkers in rapid risk stratification and prognosis for 28-day mortality in patients with sepsis in the ED.

Researchers in two other studies to date have reported Ang-2/Ang-1 ratios in patients with sepsis, and the evidence of similar Ang-1/Tie-2 ratios has been lacking. In the first study, Ricciuto et al. found that the admission levels of Ang-2/Ang-1 ratio at baseline were not robust markers for 28-day mortality in patients with severe sepsis [23]. In the second study, Wang et al. showed that the Ang-2/Ang-1 ratio was not a promising marker for severity or outcomes in pediatric patients with severe sepsis and less effective than using Ang-1 and Ang-2 alone [24]. The discrepancy between our observations and those of Ricciuto et al. may relate to the fact that Ang-2/Ang-1 ratio was measured in plasma in their study, not in serum as in ours. Also, our study population was consecutively collected and comprised a large number of subjects $(n=495)$, whereas only 70 patients with severe sepsis were enrolled in their patient cohort. Their study's small sample size might have been responsible for an underpowered or erroneous multivariable analysis lacking differences between the groups. In addition, our study population contained various degrees of early sepsis and was well divided into control $(\mathrm{n}=55)$, SIRS $(n=107)$, sepsis $(n=176)$, severe sepsis $(n=84)$, and septic shock $(n=73)$, whereas their study cohort included merely patients with severe sepsis divided into survivors $(\mathrm{n}=39)$ and nonsurvivors $(\mathrm{n}=31)$ at 28 days and did not include healthy individuals. The discrepancies between our results and those of Wang et al. may be the greatly different pathophysiology between critically ill pediatric patients and adults, along with the complex and heterogeneous nature of sepsis. Moreover, the relatively small number of pediatric patients with severe sepsis sampled $(\mathrm{n}=45)$ might have been another reason resulting in a lack of differences between the groups.

In the developed vasculature, Ang-1 protects against vascular leakage, whereas Ang-2 promotes increased vascular permeability. Ang-1 and Ang-2 appear to act as an agonist-antagonist pair mediating capillary endothelium leakage (i.e., Ang-2/Ang-1). The higher the Ang-1/ Ang-2 ratio is, the more severe the capillary endothelial damage. In addition, Ang-1 has been proposed to be necessary for the maintenance of vascular barrier function via its interaction with Tie-2 in blood vessels. As a default

Table 4 Performance of multivariable models for predicting 28-day mortality in patients with early sepsis

\begin{tabular}{llccccccc}
\hline & Variable & Cutoff & Sensitivity (\%) & Specificity (\%) & PPV (\%) & NPV (\%) & LR+ & LR- \\
\hline 28-day mortality & Ang-2/Ang-1 & 1.94 & 79.7 & 81.3 & 78.4 & 82.5 & 3.98 & 0.31 \\
& Ang-1/Tie-2 $\left(\times 10^{3}\right)$ & 30.0 & 73.0 & 82.4 & 79.4 & 77.9 & 4.15 & 0.33 \\
& PCT & 5.27 & 62.3 & 84.9 & 60.3 & 85.4 & 2.03 & 0.22 \\
& MEDS score & 14.5 & 70.6 & 76.3 & 56.2 & 90.7 & 3.35 & 0.27 \\
\hline
\end{tabular}

Abbreviations: Ang angiopoietin, $L R-$ negative likelihood ratio, $L R+$ positive likelihood ratio, MEDS Mortality in Emergency Department Sepsis, $N P V$ negative predictive value, $P C T$ procalcitonin, PPV positive predictive value, Tie-2 tyrosine kinase with immunoglobulin-like loop epidermal growth factor homology domain 2 
Table $\mathbf{5}$ Independent predictors of 28-day mortality in patients with early sepsis

\begin{tabular}{|c|c|c|c|c|c|c|c|c|c|}
\hline & \multirow[t]{2}{*}{ Variable } & \multirow[t]{2}{*}{$\beta$} & \multirow{2}{*}{$\begin{array}{l}\text { Standard } \\
\text { error }\end{array}$} & \multirow{2}{*}{$\begin{array}{l}\text { Wald } \\
\text { statistic }\end{array}$} & \multirow{2}{*}{$\begin{array}{l}\text { Degrees of } \\
\text { freedom }\end{array}$} & \multirow[t]{2}{*}{$p$ value } & \multirow{2}{*}{$\begin{array}{l}\text { Odds } \\
\text { ratio }\end{array}$} & \multicolumn{2}{|l|}{$95 \% \mathrm{Cl}$} \\
\hline & & & & & & & & Lower limit & Upper limit \\
\hline \multirow[t]{8}{*}{ 28-day mortality } & Ang-1 & -0.063 & 0.081 & 0.605 & 1 & 0.157 & 0.939 & 0.802 & 1.100 \\
\hline & Ang-2 & 0.067 & 0.022 & 8.996 & 1 & 0.003 & 1.069 & 1.023 & 1.117 \\
\hline & Tie-2 & 0.002 & 0.002 & 0.807 & 1 & 0.269 & 1.002 & 0.998 & 1.072 \\
\hline & Ang-2/Ang-1 & -0.013 & 0.043 & 0.094 & 1 & 0.026 & 0.987 & 0.909 & 1.072 \\
\hline & Ang-1/Tie-2 & -6.553 & 7.793 & 0.707 & 1 & 0.024 & 0.011 & 0.009 & 0.013 \\
\hline & $\mathrm{PCT}$ & -0.006 & 0.019 & 0.101 & 1 & 0.325 & 0.994 & 0.958 & 1.032 \\
\hline & MEDS score & 0.167 & 0.028 & 35.637 & 1 & 0.009 & 1.182 & 1.119 & 1.249 \\
\hline & Constant & 3.901 & 1.585 & 6.058 & 1 & 0.714 & 0.432 & & \\
\hline
\end{tabular}

Abbreviations: Ang angiopoietin; Cl confidence interval, MEDS Mortality in Emergency Department Sepsis, PCT procalcitonin, Tie-2 tyrosine kinase with immunoglobulinlike loop epidermal growth factor homology domain 2

pathway, constitutive Ang-1-Tie-2 interactions promote endothelial cell migration, proliferation, survival, and differentiation and regulate endothelial barrier integrity and stability [25, 26]. A lower Ang-1/Tie-2 ratio indicates a stronger defense against capillary endothelial damage. Taken together, the increase in the Ang-2/Ang-1 ratio and the decrease in the Ang-1/Tie-2 ratio demonstrate that destruction of the capillary endothelium is greater than the stability in the deterioration of sepsis. Thus, high admission serum Ang-2/Ang-1 ratio and low admission serum Ang-1/Tie-2 ratio identify the severity of sepsis in patients at the onset of early sepsis in the ED.

The Ang-2/Ang-1 and Ang-1/Tie-2 ratios were superior to the absolute Ang-1 and Ang-2 levels for prognostic purposes in critical care $[27,28]$ and had significant diagnostic and prognostic value for 28-day mortality. Our results show that an initial relative deficiency of Ang-1 level associated with a sharp increase in Ang-2 level is associated with risk stratification and clinically poorer outcome in patients with sepsis. The AUC of Ang-2/Ang-1 ratio for predicting 28-day mortality in patients with sepsis was higher than the AUCs for Ang-1 and Ang-2 levels. On the one hand, Chong and colleagues [29] recently determined that a "relative deficiency" of Ang-1 levels and a "relative adequacy" of Ang-2 levels most likely indicate the degree of capillary endothelial injury in sepsis and in patients with sepsis with high mortality arising from a more severe degree of injury to the capillary endothelium, resulting in
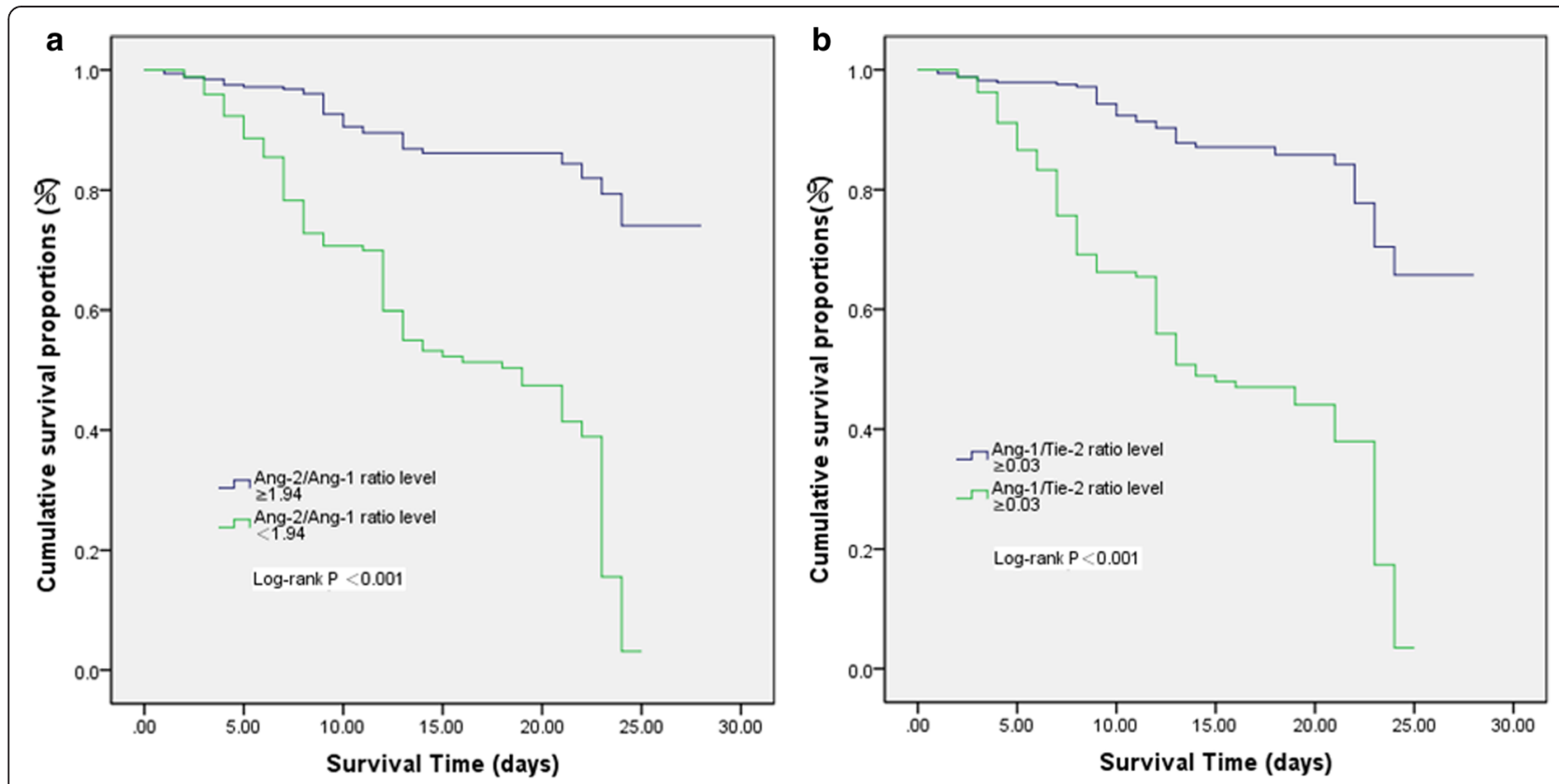

Fig. 5 a Kaplan-Meier survival curves show that patients with sepsis with Ang-2/Ang-1 ratios higher than 1.94 had a lower probability of survival at 28 days (log-rank $=94.42 ; p<0.0001)$ compared with patients with lower levels. b Similarly, patients with sepsis with Ang-1/Tie-2 ratios lower than 0.03 had a lower probability of survival at 28 days (log-rank $=100.48 ; p<0.0001$ ) than patients with lower levels. Ang angiopoietin, Tie-2 tyrosine kinase with immunoglobulin-like loop epidermal growth factor homology domain 2 
relative changes of Ang-1 and Ang-2. Thus, the ratio between these two contrasting angiogenesis factors may be capable of determining the fate of capillary endothelial cells, and Ang-2/Ang-1 ratio is likely to be a strong biomarker of sepsis mortality. On the other hand, in in vivo and vitro studies, Mofarrahi and colleagues found that lipopolysaccharide administration downregulates Ang-1 expression and functionally inhibits the Ang-1/Tie- 2 receptor pathway, resulting in an imbalance of endothelial barrier integrity and stability and subsequent organ dysfunction. This is the main reason for the use of the Ang$1 /$ Tie-2 ratio as a prognostic biomarker of sepsis [26].

Currently, PCT is the most studied and promising sepsis biomarker for diagnosis, risk stratification, evaluation of prognosis, and therapy monitoring [30]. Previous studies of the utility of PCT have been focused mainly on their diagnostic value for bacterial infection and are not a specific indicator of all types of infection in sepsis [31]. A substantial number of studies have challenged the prognostic role of PCT [32]. In addition, the MEDS score is more suitable than the other severity scoring systems for predicting mortality among patients with sepsis in the ED, despite the controversial value of clinical application [33]. In our study, the PCT level and MEDS score were chosen as a variable to compare with the Ang-2/Ang-1 and Ang-1/Tie-2 ratios.

Because there are advantages and disadvantages of biomarkers and scoring systems in the prognosis of sepsis, the combination between biomarkers with severity scoring systems can overcome their limitations and improve the accuracy of prognosis. In our study, the combination of the Ang-2/Ang-1 and Ang-1/Tie-2 ratios with the MEDS score was more effective than that of PCT or the MEDS score alone in predicting 28-day mortality. PCT was used merely to support the diagnosis of bacterial infection, but it exhibited no discriminative power for the prediction of mortality in early sepsis. Although the MEDS score is regarded as an easy and reliable method for predicting mortality in patients with a suspected infection, the discriminative ability of the combination of PCT and the MEDS score in the prognostic evaluation of sepsis is still limited. However, in a more comprehensive aspect, the combination of the Ang-2/ Ang-1 and Ang-1/Tie-2 ratios with the MEDS score indicates the degree of general capillary endothelial damage and pathological condition of patients with sepsis and reflects the pathophysiological mechanism of widespread capillary endothelium and the risk of short-term mortality of patients in early sepsis. Taken together, the Ang-2/Ang-1 and Ang-1/Tie-2 ratios enhanced the accuracy of PCT and the MEDS score in prognostic evaluation.

In addition, there were significant close correlations between the Ang-1, Ang-2, and Tie-2 levels [6] and between the Ang-2/Ang-1 or Ang-1/Tie-2 ratios and the
MEDS score. Similar associations between the Ang-2/ Ang-1 or Ang-1/Tie-2 ratios and the MEDS score in critically ill patients have not been reported previously. The survival curves clearly illustrated the optimized information provided by the use of the Ang-2/Ang-1 or Ang-1/Tie-2 ratios. Both markers above or below their respective cutoff value indicate a high risk of death.

\section{Limitations}

Some limitations of this study merit consideration. First, convenience sampling or the subjective inclusion criterion of "suspicion of infection" may have introduced bias into the clinical portion of this work. Second, the patient information on pathogenic evidence and treatment preferences was not considered in the initial study. Third, comparisons of the Ang-1, Ang-2, and Tie-2 levels before and after treatment were not performed during the patients' stay in the ED. Fourth, the complicated underlying diseases might have had an effect on the results.

\section{Conclusions}

Serum Ang-2/Ang-1 and Ang-1/Tie-2 ratios are independent predictors of 28-day mortality in patients with sepsis in the ED. The serum Ang-2/Ang-1 and Ang-1/Tie2 ratios are associated with risk stratification and prognostic evaluation of sepsis and are more valuable predictors of 28-day mortality than PCT and MEDS score.

\section{Key messages}

- The serum Ang-2/Ang-1 and Ang-1/Tie-2 ratios were good biomarkers for reflecting the severity of sepsis.

- The serum Ang-2/Ang-1 and Ang-1/Tie-2 ratios were superior to PCT and MEDS score for predicting 28-day mortality in patients with sepsis in the ED.

- The serum Ang-2/Ang-1 and Ang-1/Tie-2 ratios and MEDS score were all independent predictors of 28-day mortality in patients with sepsis.

- The serum Ang-2/Ang-1 or Ang-1/Tie-2 ratios, in combination with PCT and MEDS score, enhanced the predictive accuracy of 28-day mortality in patients with sepsis.

\section{Abbreviations}

AECOPD: acute exacerbations of chronic obstructive pulmonary disease; Ang: angiopoietin; APACHE: Acute Physiology and Chronic Health Evaluation; AUC: area under the receiver operating characteristic curve; $\mathrm{Cl}$ : confidence interval; CONSORT: Consolidated Standards of Reporting Trials; DKA: diabetic ketoacidosis; ED: emergency department; HR: heart rate; IAl: intraabdominal infection; LR-: negative likelihood ratio; LR+: positive likelihood ratio; MAP: mean arterial pressure; MEDS: Mortality in Emergency Department Sepsis; MODS: multiple organ dysfunction syndrome; NPV: negative predictive value; OR: odds ratio; $\mathrm{PaO}_{2} / \mathrm{FiO}_{2}$ : ratio of partial pressure arterial oxygen to fraction of inspired oxygen; PCT: procalcitonin; PE: pulmonary embolism; PPV: positive predictive value; RI: respiratory infection; ROC: receiver operating characteristic; SD: standard deviation; SIRS: systemic inflammatory response syndrome; Tie-2: tyrosine kinase with

immunoglobulin-like loop epidermal growth factor homology domain 2. 


\section{Competing interests}

The authors declare that they have no competing interests.

\section{Authors' contributions}

YF conducted the trial, collected data, and drafted and revised the manuscript for important intellectual content. CL took responsible for the overall content as the guarantor and helped to revise the manuscript. RS conceived of the study, collected data, and helped to draft the manuscript. HY collected data and helped to draft the manuscript. QZ participated in study design, collected data, and helped to draft the manuscript. LZ analyzed the data, performed the statistical analysis, and helped to revise the manuscript. All authors read and approved the final manuscript.

\section{Acknowledgments}

The authors gratefully thank Chen Yunxia and Liu Bo for excellent assistance concerning statistical analysis, and they also thank the staff of both the ED and local medical research centers for their excellent and skillful guidance.

\section{Received: 24 June 2015 Accepted: 22 September 2015}

Published online: 14 October 2015

\section{References}

1. Lever A, Mackenzie I. Sepsis: definition, epidemiology, and diagnosis. BMJ. 2007;335:879-83.

2. Martin GS, Mannino DM, Eaton S, Moss M. The epidemiology of sepsis in the United States from 1979 through 2000. N Engl J Med. 2003;348:1546-54

3. Levy MM, Fink MP, Marshall JC, Abraham E, Angus D, Cook D, et al. 2001 SCCM/ESICM/ACCP/ATS/SIS International Sepsis Definitions Conference. Crit Care Med. 2003;31:1250-6.

4. Aird WC. The role of the endothelium in severe sepsis and multiple organ dysfunction syndrome. Blood. 2003;101:3765-77.

5. Peters K, Unger RE, Brunner J, Kirkpatrick CJ. Molecular basis of endothelial dysfunction in sepsis. Cardiovasc Res. 2003;60:49-57.

6. Fiedler $\mathrm{U}$, Augustin HG. Angiopoietins: a link between angiogenesis and inflammation. Trends Immunol. 2006;27:552-8.

7. Witzenbichler B, Westermann D, Knueppel S, Schultheiss HP, Tschope C. Protective role of angiopoietin-1 in endotoxic shock. Circulation. 2005;111:97-105

8. Thurston G, Rudge JS, loffe E, Zhou H, Ross L, Croll SD, et al. Angiopoietin-1 protects the adult vasculature against plasma leakage. Nat Med. 2000;6:460-3.

9. Fiedler U, Reiss Y, Scharpfenecker M, Grunow V, Koidl S, Thurston G, et al Angiopoietin-2 sensitizes endothelial cells to TNF-a and has a crucial role in the induction of inflammation. Nat Med. 2006;12:235-9.

10. Parikh SM, Mammoto T, Schultz A, Yuan HT, Christiani D, Karumanchi SA, et al. Excess circulating angiopoietin-2 may contribute to pulmonary vascular leak in sepsis in humans. PLoS Med. 2006;3:e46.

11. Wong AL, Haroon ZA, Werner S, Dewhirst MW, Greenberg CS, Peters KG. Tie2 expression and phosphorylation in angiogenic and quiescent adult tissues. Circ Res. 1997;81:567-74.

12. Tadros A, Hughes DP, Dunmore BJ, Brindle NP. ABIN-2 protects endothelial cells from death and has a role in the antiapoptotic effect of angiopoietin-1. Blood. 2003;102:4407-9.

13. Siner JM, Bhandari V, Engle KM, Elias JA, Siegel MD. Elevated serum angiopoietin 2 levels are associated with increased mortality in sepsis. Shock. 2009;31:348-53.

14. Huang YQ, Sauthoff H, Herscovici P, Pipiya T, Cheng J, Heitner S, et al. Angiopoietin-1 increases survival and reduces the development of lung edema induced by endotoxin administration in a murine model of acute lung injury. Crit Care Med. 2008;36:262-7.

15. Lymperopoulou K, Velissaris D, Kotsaki A, Antypa E, Georgiadou S, Tsaganos $T$, et al. Angiopoietin-2 associations with the underlying infection and sepsis severity. Cytokine. 2015;73:163-8.

16. David S, Mukherjee A, Ghosh CC, Yano M, Khankin EV, Wenger JB, et al. Angiopoietin-2 may contribute to multiple organ dysfunction and death in sepsis. Crit Care Med. 2012;40:3034-41.

17. Levy MM, Fink MP, Marshall JC, Abraham E, Angus D, Cook D, et al. 2001 SCCM/ESICM/ACCP/ATS/SIS International Sepsis Definitions Conference. Intensive Care Med. 2003:29:530-8.

18. Dellinger RP, Levy MM, Rhodes A, Annane D, Gerlach H, Opal SM, et al. Surviving Sepsis Campaign: international guidelines for management of severe sepsis and septic shock, 2012. Intensive Care Med. 2013;39:165-228.
19. Shapiro NI, Wolfe RE, Moore RB, Smith E, Burdick E, Bates DW. Mortality in Emergency Department Sepsis (MEDS) score: a prospectively derived and validated clinical prediction rule. Crit Care Med. 2003;31:670-5.

20. Knaus WA, Draper EA, Wagner DP, Zimmerman JE. APACHE II: a severity of disease classification system. Crit Care Med. 1985;13:818-29.

21. Fluss R, Faraggi $D$, Reiser $B$. Estimation of the Youden Index and its associated cutoff point. Biom J. 2005;47:458-72.

22. Siner JM. A tale of two ligands: angiopoietins, the endothelium, and outcomes. Crit Care. 2013;17:1007.

23. Ricciuto DR, dos Santos CC, Hawkes M, Toltl L, Conroy AL, Rajwans N, et al. Angiopoietin-1 and angiopoietin-2 as clinically informative prognostic biomarkers of morbidity and mortality in severe sepsis. Crit Care Med. 2011:39:702-10.

24. Wang K, Bhandari V, Giuliano Jr JS, O'Hern CS, Shattuck MD, Kirby M. Angiopoietin-1, angiopoietin-2 and bicarbonate as diagnostic biomarkers in children with severe sepsis. PLoS One. 2014;9:e108461.

25. Alves BE, Montalvao SA, Aranha FJ, Siegl TF, Souza CA, Lorand-Metze I, et al. Imbalances in serum angiopoietin concentrations are early predictors of septic shock development in patients with post chemotherapy febrile neutropenia. BMC Infect Dis. 2010;10:143.

26. Mofarrahi M, Nouh T, Qureshi S, Guillot L, Mayaki D, Hussain SN. Regulation of angiopoietin expression by bacterial lipopolysaccharide. Am J Physiol Lung Cell Mol Physiol. 2008;294:L955-63.

27. Brindle NP, Saharinen P, Alitalo K. Signaling and functions of angiopoietin-1 in vascular protection. Circ Res. 2006;98:1014-23.

28. Giuliano Jr JS, Lahni PM, Harmon K, Wong HR, Doughty LA, Carcillo JA, et al. Admission angiopoietin levels in children with septic shock. Shock. 2007:28:650-4.

29. Chong AY, Caine GJ, Freestone B, Blann AD, Lip GY. Plasma angiopoietin-1, angiopoietin-2, and angiopoietin receptor tie-2 levels in congestive heart failure. J Am Coll Cardiol. 2004;43:423-8.

30. Lichtenstern C, Brenner T, Bardenheuer HJ, Weigand MA. Predictors of survival in sepsis: what is the best inflammatory marker to measure? Curr Opin Infect Dis. 2012;25:328-36.

31. Simon L, Gauvin F, Amre DK, Saint-Louis P, Lacroix J. Serum procalcitonin and C-reactive protein levels as markers of bacterial infection: a systematic review and meta-analysis. Clin Infect Dis. 2004;39:206-17.

32. Dahaba AA, Hagara B, Fall A, Rehak PH, List WF, Metzler H. Procalcitonin for early prediction of survival outcome in postoperative critically ill patients with severe sepsis. Br J Anaesth. 2006;97:503-8.

33. Howell MD, Donnino MW, Talmor D, Clardy P, Ngo L, Shapiro NI. Performance of severity of illness scoring systems in emergency department patients with infection. Acad Emerg Med. 2007;14:709-14.

\section{Submit your next manuscript to BioMed Central and take full advantage of:}

- Convenient online submission

- Thorough peer review

- No space constraints or color figure charges

- Immediate publication on acceptance

- Inclusion in PubMed, CAS, Scopus and Google Scholar

- Research which is freely available for redistribution 